\title{
ON THE NUMERICAL SOLUTION OF THE DIFFUSION EQUATION SUBJECT TO THE SPECIFICATION OF MASS
}

\author{
A. B. GUMEL ${ }^{1}$ \\ (Received 25 February 1997; revised 9 September 1997)
}

\begin{abstract}
A parallel algorithm is developed for the numerical solution of the diffusion equation $u_{t}=u_{x x}, 0<x<X, 0<t<T$, subject to $u(x, 0)=f(x), u_{x}(X, t)=g(t)$ and the specification of mass $\int_{a}^{b} u(x, t) d x=M(t), 0<b<X$.
\end{abstract}

\section{Introduction}

It is known [2] that because certain chemicals absorb light at various frequencies, the intensity of such light on a photoelectric cell gives an electric signal which is proportional to the total amount of chemical present in the volume through which the light passes. Suppose $u(x, t)$ denotes the concentration of such a chemical which is diffusing in a straight tube with $x$ measured in the direction of the axis of the tube. Then the electric signal produced by a light beam passing through the tube at right angles between $x=0$ and $x=b$ is proportional to $\int_{0}^{b} u(x, t) d x$. This integral represents the total mass of the chemical in $0 \leq x \leq b$ at time $t$.

This paper considers the problem of obtaining numerical approximations to the concentration $u=u(x, t)$ which satisfies the partial differential equation (PDE)

$$
\frac{\partial u}{\partial t}=\frac{\partial^{2} u}{\partial x^{2}}, \quad 0<x<X, t>0,
$$

subject to a Neumann time-dependent boundary condition on the boundary $\partial \Omega$ of the open-region $\Omega$ defined by the lines $x=0, x=X$ given by

$$
\frac{\partial u(X, t)}{\partial x}=g(t), \quad 0 \leq t \leq T,
$$

${ }^{1}$ Faculty of Information Technology, University Telekom, 75450 Melaka, Malaysia.

(C) Australian Mathematical Society, 1999, Serial-fee code 0334-2700/99 
and the specification of mass

$$
\int_{0}^{b} u(x, t) d x=M(t), \quad 0<b<X,
$$

with initial conditions

$$
u(x, 0)=f(x), \quad 0<x<X
$$

where $f, g$ and $M$ are known functions and are assumed to be sufficiently smooth to produce a smooth classical solution of $u$. The existence and uniqueness properties of the solution of this problem are detailed in [1]. A number of sequential numerical procedures have been suggested in the literature for the solution of this problem; see, for instance, [2, 3].

In the present paper, the method of lines semi-discretization approach will be used to transform the model PDE into a system of first-order, linear, ordinary differential equations (ODEs), the solution of which satisfies a certain recurrence relation involving matrix exponential terms. A suitable rational approximant will be used to approximate such exponentials leading to an $L_{0}$-stable algorithm which may be parallelized through a partial-fraction splitting technique. These $L_{0}$-stable schemes, unlike $A_{0}$-stable schemes, are known to be suitable for use in integrating PDEs with time-dependent boundary conditions in which discontinuities between initial conditions and boundary conditions (such as the test problems to be considered in this paper) exist $[4,10,11]$.

\section{Discretization and recurrence relation}

The interval $0 \leq x \leq X$ is divided into $N+1$ subintervals each of width $h$, so that $(N+1) h=X$ and the time variable $t$ is discretized in steps of length $\ell$. Thus at each time level $t=t_{n}=n \ell(n=0,1,2 \ldots)$, the open region $\Omega=[0<x<X] \times[t>0]$ and its boundary $\partial \Omega$ consisting of the lines $x=0$ and $x=X$ and the axis $t=0$ have been superimposed by a rectangular mesh with $N$ points within $\Omega$ and one point along each side of $\partial \Omega$.

The solution $u(x, t)$ of (1) is sought at each point $(k h, n \ell)$ in $\Omega \times[t>0]$, where $k=1,2, \ldots, N$ and $n=0,1,2 \ldots$ The solution of an approximating numerical method will be denoted by $U(x, t)$. The space derivatives in (1) and (2) will be replaced by their second-order central-difference approximants given by

$$
\left.\frac{\partial^{2} u}{\partial x^{2}}=h^{-2}[u(x-h), t)-2 u(x, t)+u(x+h, t)\right]+O\left(h^{2}\right) \text { as } h \rightarrow 0
$$


and

$$
\frac{\partial u}{\partial x}=\frac{u(x+h, t)-u(x-h, t)}{2 h}+O\left(h^{2}\right) \text { as } h \rightarrow 0, \text { respectively. }
$$

\section{Treatment of the non-local boundary condition}

The integral in (3) may be approximated using a quadrature rule such as Simpson's rule to give

$$
\int_{0}^{b} u(x, t) d x \approx \frac{h^{*}}{3}\left[u(0, t)+4 \sum_{i=1}^{\frac{1}{2}} u(2 i-1, t)+2 \sum_{i=1}^{\frac{1}{2}-1} u(2 i, t)+u(J, t)\right]+O\left(h^{4}\right)
$$

in which $h^{*}=b / J$. Thus, the boundary conditions $u(1, t)$ and $u(0, t)$ may then be determined using (2) with (6) and (3) with (7) respectively.

Applying (1) to all the interior mesh points within $\Omega$ at time level $t_{n}=n \ell$, with the space derivative replaced by (5), leads to a system of $N$ first-order, linear, ordinary differential equations of the form

$$
\frac{d \mathbf{U}(t)}{d t}=A \mathbf{U}(t)+\psi(t), \quad t>0, \mathbf{U}(0)=\mathbf{f}
$$

in which the matrix $A$ is of order $N$ and is given by

$$
A=h^{-2}\left(\begin{array}{cccccc}
\alpha_{1} & \alpha_{2} & \alpha_{3} & \cdots \alpha_{J} & & 0 \\
1 & -2 & 1 & & & \\
& \ddots & \ddots & \ddots & & \\
& & 1 & -2 & 1 & \\
0 & & & & 2 & -2
\end{array}\right) \text {, }
$$

where $\alpha_{1}=-6, \alpha_{2}=-1$ and $\alpha_{i}= \begin{cases}-4 & \text { for } i=3(2) J-1 \\ -2 & \text { for } i=4(2) J-2 \\ -1 & \text { for } i=J\end{cases}$

In (8), the vector $\psi(t)$ arises from the use of the boundary conditions $u(0, t)$ and $u(1, t)$ in (5).

Solving the system of ODEs (8) subject to the initial condition $U(0)=\mathbf{f}$, gives $[11$, p. 136],

$$
\mathbf{U}(t)=\exp (t A) \mathbf{f}+\int_{0}^{t} \exp [(t-s) A] \psi(s) d s, \quad t \geq 0,
$$

which satisfies the recurrence relation

$$
\mathbf{U}(t+\ell)=\exp (\ell A) \mathbf{U}(t)+\int_{t}^{t+\ell} \exp [(t+\ell-s) A] \psi(s) d s, \quad t=0, \ell, 2 \ell, \ldots
$$




\section{The parallel algorithm}

The integral in (11) may be approximated by a quadrature formula of the form

$$
\int_{t}^{t+\ell} \exp [(t+\ell-k) A] \psi(k) d k \approx W_{1} \psi\left(k_{1}\right)+W_{2} \psi\left(k_{2}\right), \quad k_{1} \neq k_{2},
$$

in which the weights $W_{1}=W_{1}(\ell A)$ and $W_{2}=W_{2}(\ell A)$. Following Lawson [7] and Swayne [9], the vector $\psi(k)$ in (12) is successively given the values $\psi(k) \equiv$ $[1,1, \ldots, 1]^{\top}$ and $\psi(k) \equiv[k, k, \ldots, k]^{\top}$ (with $\mathrm{T}$ denoting transpose) to give

$$
\begin{aligned}
W_{1}+W_{2} & =Q_{1}, \\
k_{1} W_{1}+k_{2} W_{2} & =Q_{2},
\end{aligned}
$$

in which $Q_{1}=Q_{1}(\ell A)=A^{-1} \exp (\ell A)-A^{-1}$ and $Q_{2}=Q_{2}(\ell A)=A^{-1}[-(t+\ell) I+$ $\left.t \exp (\ell A)-A^{-1}+A^{-1} \exp (\ell A)\right]$, where $I$ is the identity matrix of order $N$. Solving (13) for distinct abscissae $k_{1}$ and $k_{2}$ gives

$$
W_{1}=\frac{k_{2} Q_{1}-Q_{2}}{k_{2}-k_{1}} \quad \text { and } \quad W_{2}=\frac{k_{1} Q_{1}-Q_{2}}{k_{1}-k_{2}} \text {. }
$$

Substituting (13) and (14) into (12) and using $k_{1}=t$ and $k_{2}=t+\ell$ leads to the recurrence relation

$$
\begin{aligned}
\mathrm{U}(t+\ell)= & \exp (\ell A) \mathrm{U}(t)+(\ell A)^{-1}\left[\ell \exp (\ell A)+A^{-1}-A^{-1} \exp (\ell A)\right] \psi(t) \\
& +(\ell A)^{-1}\left[A^{-1} \exp (\ell A)-\ell I-A^{-1}\right] \psi(t+\ell) ; t=0, \ell, 2 \ell, \ldots
\end{aligned}
$$

The development of numerical methods will be based on making appropriate approximations to the exponentials in this recurrence relation. Higher-order Padé approximants [8] are popularly employed (see, for instance, $[4,5,11]$ ) for such exponentials. Methods based on the use of these approximants are of high accuracy in time and, in the case of the subdiagonal Padés, have good stability properties. However, a major drawback associated with the efficient implementation of these methods is that complex arithmetic is required in the numerical computation. This is because the poles of the higher-order Padés occur in complex conjugate pairs.

In order to circumvent this drawback, a second-order rational approximant given by [4]

$$
R(\ell A) \approx\left(I-e \ell A+\left(e-\frac{1}{2}\right) \ell^{2} A^{2}\right)^{-1}[I+(1-e) \ell A],
$$

with $e \approx 0.54$, will be used for the matrix exponentials in the recurrence relation (15). A useful feature of $R(\ell A)$ is that its denominator has real distinct poles thus 
guaranteeing the use of real arithmetic (only) in the computation. It is easy to see that the numerical method resulting from the use of (16) in (15) is second-order accurate. Assuming the eigenvalues of the matrix $A$ are real and have negative real parts, a linearised stability analysis of the von Neumann type suggests that the numerical method arising from the use of (16) in (15) is $L_{0}$-stable. The merits of these schemes over $A_{0}$-stable methods such as the Crank-Nicholson method (arising from the use of the $(1,1)$ Padé approximant in $(18)$ ) and the Peaceman-Rachford method for solving problems with discontinuities between initial conditions and boundary conditions are emphasized in [6].

Using (16) in (15) gives

$$
\mathbf{U}(t+\ell)=R(\ell A) \mathbf{U}(t)+\frac{\ell}{2}[S(\ell A) \psi(t)+T(\ell A) \psi(t+\ell)] ; \quad t=0, \ell, 2 \ell, \ldots,
$$

in which

$$
S(\ell A)=\left[1-e \ell A+\left(e-\frac{1}{2}\right) \ell^{2} A^{2}\right]^{-1}
$$

and

$$
T(\ell A)=\left[I-e \ell A+\left(e-\frac{1}{2}\right) \ell^{2} A^{2}\right]^{-1}\left[I-2\left(e-\frac{1}{2}\right) \ell A\right] .
$$

In order to implement (17) in parallel, the functions $R(\ell A), S(\ell A)$ and $T(\ell A)$ are decomposed into their partial-fraction forms given by

$$
\begin{aligned}
& R(\ell A)=s_{1}\left(I-r_{1} \ell A\right)^{-1}+s_{2}\left(I-r_{2} \ell A\right)^{-1}, \\
& S(\ell A)=s_{3}\left(I-r_{1} \ell A\right)^{-1}+s_{4}\left(I-r_{2} \ell A\right)^{-1}, \\
& T(\ell A)=s_{5}\left(I-r_{1} \ell A\right)^{-1}+s_{6}\left(I-r_{2} \ell A\right)^{-1},
\end{aligned}
$$

with

$$
\begin{aligned}
s_{1} & =\frac{1-e+r_{1}}{r_{1}-r_{2}}, & s_{2} & =\frac{1-e+r_{2}}{r_{2}-r_{1}}, \\
s_{3} & =\frac{r_{1}}{r_{1}-r_{2}}, & s_{4} & =\frac{r_{2}}{r_{2}-r_{1}}
\end{aligned}
$$

and

$$
s_{5}=\frac{1-2 e+r_{1}}{r_{1}-r_{2}}, \quad s_{6}=\frac{1-2 e+r_{2}}{r_{2}-r_{1}},
$$


where

$$
r_{1}=\frac{2 e-1}{e+\sqrt{\left(e^{2}-4 e+2\right)}}, \quad r_{2}=\frac{2 e-1}{e-\sqrt{\left(e^{2}-4 e+2\right)}} .
$$

The solution vector $\mathrm{U}(t+\ell)$ in (17) may now be obtained in parallel using two processors running concurrently as follows:

$$
\text { Processor 1: } \begin{aligned}
\left(I-r_{1} \ell A\right) \mathbf{z}_{1} & =s_{1} \mathbf{U}(t), \\
& \left(I-r_{1} \ell A\right) \mathbf{z}_{3}=s_{3} \psi(t), \\
& \left(I-r_{1} \ell A\right) \mathbf{z}_{5}=s_{5} \psi(t+\ell), \\
\text { Processor 2: } \quad\left(I-r_{2} \ell A\right) \mathbf{z}_{2} & =s_{2} \mathbf{U}(t), \\
\left(I-r_{2} \ell A\right) \mathbf{z}_{4} & =s_{4} \psi(\mathbf{t}), \\
\left(I-r_{2} \ell A\right) \mathbf{z}_{6} & =s_{6} \psi(\mathbf{t}+\ell), \\
\text { Then } \mathbf{U}(t+\ell) & =\mathbf{z}_{1}+\mathbf{z}_{2}+\frac{\ell}{2}\left[\mathbf{z}_{3}+\mathbf{z}_{4}+\mathbf{z}_{5}+\mathbf{z}_{6}\right] .
\end{aligned}
$$

This algorithm is denoted by PRL. The intermediate vectors $\mathbf{z}_{i}(i=1, \ldots, 6)$ need not be stored once $\mathbf{U}(t+\ell)$ is computed at each time step. The coefficient matrices $\left(I-r_{1} \ell A\right)$ and $\left(I-r_{2} \ell A\right)$ are decomposed into lower and upper (LU) triangular forms only once. These LU products are then "fed" to the two processors in order to compute the intermediate vectors $\mathbf{z}_{i}(i=1, \ldots, 6)$ using forward and backward substitutions. In the case of the simple heat equation, $\psi \equiv 0$ and thus $\mathbf{z}_{3}=\mathbf{z}_{4}=\mathbf{z}_{5}=\mathbf{z}_{6}=0$ in (27).

\section{Numerical experiments}

In order to test the behaviour of the $L_{0}$-stable scheme (17), four problems from the literature were considered. Comparisons were made, in terms of accuracy, with the discrete Galerkin (DG) scheme developed in [2] and the finite difference scheme DFD reported in [3].

PROBLEM 1. Following Cannon et al. [3], the PDE (1) subject to (2) and (3) with

$$
\begin{aligned}
f(x) & =\frac{1}{2} x^{2}, \\
g(t) & =1, \\
M(t) & =0.75 t+\frac{1}{6}(0.75)^{3}
\end{aligned}
$$

and with theoretical solution

$$
u(x, t)=\frac{1}{2} x^{2}+t
$$


TABLE 1. Relative errors at various time lengths with $h=\ell=0.0025$.

\begin{tabular}{|c|c|c|c|c|}
\hline Time length & DG & DFD & PRL & Theoretical Solution \\
\hline$t=0.01$ & $1.93 E-3$ & $2.40 E-5$ & $1.61 E-6$ & $4.125 E-2$ \\
\hline$t=0.025$ & $9.93 E-4$ & $1.27 E-4$ & $8.81 E-5$ & $5.625 E-2$ \\
\hline$t=0.1$ & $1.8 E-3$ & $4.93 E-4$ & $8.07 E-4$ & 0.13125 \\
\hline
\end{tabular}

is solved. The absolute relative errors $|(u-U) / u|$ computed at various time lengths with $h=0.0025$ and time-step $\ell=0.0025$ are tabulated in Table 1 .

PROBLEM 2. Here

$$
\begin{aligned}
f(x) & =\cos (x), \\
g(t) & =-\exp (-t) \sin (1.0), \\
M(t) & =\exp (-t) \sin (0.75),
\end{aligned}
$$

with theoretical solutions

$$
u(x, t)=\exp (-t) \cos (x)
$$

TABLE 2. Relative errors at various time lengths with $h=\ell=0.0025$.

\begin{tabular}{|c|c|c|c|c|}
\hline Time length & DG & DFD & PRL & Theoretical Solution \\
\hline$t=0.01$ & $8.90 E-5$ & $2.75 E-5$ & $3.73 E-8$ & 0.9593 \\
\hline$t=0.025$ & $6.00 E-5$ & $1.05 E-4$ & $2.82 E-6$ & 0.9450 \\
\hline$t=0.1$ & $2.81 E-4$ & $5.27 E-4$ & $6.29 E-5$ & 0.8767 \\
\hline
\end{tabular}

Table 2 contains the absolute relative errors at various time lengths using $h=\ell=$ 0.0025 .

PROBLEM 3. In this experiment,

$$
\begin{aligned}
f(x) & =\sin (\pi x), \\
g(t) & =-\pi \exp \left(-\pi^{2} t\right), \\
M(t) & =\frac{1}{\pi}\left(\frac{1}{\sqrt{2}}+1\right) \exp \left(-\pi^{2} t\right)
\end{aligned}
$$

and the theoretical solution is

$$
u(x, t)=\exp \left(-\pi^{2} t\right) \sin (\pi x) .
$$

The computed results at various time lengths with $h=\ell=0.0025$ are shown in Table 3. 
TABLE 3. Relative errors at various time lengths with $h=\ell=0.0025$.

\begin{tabular}{|c|c|c|c|c|}
\hline Time length & DG & DFD & PRL & Theoretical Solution \\
\hline$t=0.01$ & $4.00 E-6$ & $2.44 E-5$ & $2.95 E-6$ & 0.6407 \\
\hline$t=0.025$ & $3.00 E-6$ & $1.06 E-4$ & $2.81 E-7$ & 0.5525 \\
\hline$t=0.1$ & $7.00 E-6$ & $8.36 E-4$ & $3.55 E-5$ & 0.2635 \\
\hline
\end{tabular}

PROBLEM 4. Following Cannon et al. [3], the effect of time-steps on the numerical method (27) is investigated by solving Problem 3 with three different time-steps, namely $\ell=0.01, \ell=0.05$, and $\ell=0.025$. The relative errors at $t=0.1$ are given in Table 4.

TABLE 4. Relative errors at $t=0.1$ with $h=0.0025$ using various time-steps.

\begin{tabular}{|c|c|c|c|c|}
\hline Time step & DG & DFD & PRL & Theoretical Solution \\
\hline$\ell=0.01$ & $1.33 E-4$ & $1.51 E-3$ & $3.99 E-4$ & 0.2635 \\
\hline$\ell=0.05$ & $1.63 E-2$ & $1.13 E-4$ & $1.28 E-2$ & 0.2635 \\
\hline$\ell=0.025$ & - & $8.36 E-4$ & $3.55 E-5$ & 0.2635 \\
\hline
\end{tabular}

Clearly, discontinuities between initial conditions and boundary conditions exist in all the four problems. Tables 1 to 4 confirm that the $L_{0}$-stable scheme (27) is very accurate and thus that it is suited for solving problems of this nature.

\section{Conclusion}

An $O\left(h^{2}+\ell^{2}\right) L_{0}$-stable parallel algorithm has been developed for the simple heat equation with non-local boundary specifications. The algorithm was found to be more accurate in comparison with two existing algorithms from the literature, and may be implemented in parallel using a machine with two processors.

\section{References}

[1] J. R. Cannon and J. van der Hoek, "The existence of and a continuous dependence result for the solution of the heat equation subject to the specification of energy", Suppl. Bolletino Unione Mat. Ital. 1 (1981) 253-282.

[2] J. R. Cannon, S. P. Esteva and J. van der Hoek, "A Galerkin procedure for the diffusion equation subject to the specification of mass", SIAM J. Numer. Anal. 24 (1987) 499-515.

[3] J. R. Cannon, Yanping Lin and Shingmin Wang, "An implicit finite difference scheme for the diffusion equation subject to mass specification", Int. J. Engng. Sci. 28 (7) (1990) 573-578. 
[4] A. B. Gumel, "Parallel and sequential algorithms for parabolic equations with applications", (PhD Thesis, Brunel University, 1993).

[5] A. B. Gumel, E. H. Twizell and M. A. Arigu, "Parallel $L_{0}$-stable methods for multi-dimensional heat equation", Parallel Algorithms and Applications 11 (1997) 13-25.

[6] J. D. Lambert, Numerical methods for ordinary differential systems: The initial-value problem (John Wiley and Sons, Chichester, 1991).

[7] J. D. Lawson, "Some numerical methods for stiff ordinary and partial differential equations", Second Manitoba Conf. on Numerical Mathematics, Winnipeg, Canada (1972) 27-34.

[8] M. H. Padé, "Sur la répresentation approcheé d'une fonction par des fractions rationelles", Ann. de l'Ecole Normale Supérieure 9 (suppl) (1982).

[9] D. A. Swayne, "Time-dependent boundary and interior forcing in locally one-dimensional schemes", SIAM J. Sci. Stat. Comput. 8 (1987) 755-767.

[10] E. H. Twizell, Numerical methods, with applications in the biomedical sciences (Ellis Horwood, Chichester, and John Wiley, New York, 1988).

[11] E. H. Twizell, A. Q. M. Khaliq and D. A. Voss, "Sequential and parallel algorithms for second-order initial-value problems", WSSIA A 2 (1993) 399-412. 